\title{
Review of: "Spatiotemporal tracing of pandemic spread from infection data"
}

nidhi manaktala ${ }^{1}$

1 Manipal University

Potential competing interests: The author(s) declared that no potential competing interests exist.

It seems to be a well written article. 